\title{
Sleep, circadian rhythms, and interval timing: evolutionary strategies to time information
}

\author{
ValterTucci* \\ Department of Neuroscience and Brain Technologies, Istituto Italiano di Tecnologia, Genova, Italy \\ ${ }^{*}$ Correspondence: valter.tucci@iit.it
}

A crucial property of the brain is to integrate temporal information with accurate physiological responses (Hinton and Meck, 1997; Buhusi and Meck, 2005; Coull et al., 2011). Evolution has favored biological clocks that dictate homeostatic processes (e.g., the circadian timing of sleep) and, on a smaller time-scale, timed behavioral responses (e.g., interval timing). The interplay between such time-keeping mechanisms is intriguing but biologically complex. Moreover, in biology, analogous problems can be successfully solved by multiple computations. In this article I will discuss of sleep, circadian rhythms, and interval timing by delineating several aspects that suggest a common evolutionary role in providing neurobiological mechanisms for temporal information processing.

Neither interval timing nor the homeostatic regulation of sleep are currently as well-understood, at the molecular level, as the circadian clock. This has triggered a scientific interest in linking these phenomena to the circadian molecular machinery. On one hand, sleep homeostasis has been investigated in genetic and lesion studies of the circadian "master" clock (Franken and Dijk, 2009) to test whether the two processes (homeostatic and circadian) were independent. On the other hand, it has been questioned whether interval timing and circadian clock share similar oscillatory mechanisms, although with a different time-scale (Crystal, 2001, 2006a,b; Crystal and Baramidze, 2007), and whether circadian rhythms affect interval timing. Whilst the research in sleep and circadian clock over the last few years resulted in some interesting positive associations (reviewed in Tucci and Nolan, 2010), the question whether interval timing is related to the circadian clock has not a clear unanimous conclusion yet.

\section{SLEEP AND INTERVAL TIMING}

As in conditioning behaviors, in which a brief time interval itself may embody the proper information to be learned, sleep behavior is phase-locked in the 24-h (circadian) rhythms of the organism. However, while the circadian process is self-sustained, sleep is homeostatically regulated and varies according to the previous wakefulness. Back in evolution, single-cell organisms became entrained to environmental rest-activity stimuli (light and temperature changes caused by the earth's rotation) in order to set the time of their metabolic processes (Krueger, 2010). Sleep evolved from restactivity cycles and it is still debated whether a sleep-like phenotype occurs within single neurons and how this is affected by environmental (extracellular) stimuli. Nevertheless, it is reasonable to envisage that sleep has developed, in multicellular organisms, based on the same biology of a primordial rest state.

In understanding the brain mechanisms of interval timing it is becoming pivotal to define whether the coding of interval timing depends on targeted neuronal mechanisms and their associated sub-cellular signaling pathways or on the states of neuronal networks. There is no doubt that the two mechanisms ( single neuronal signaling and networks) are intimately connected but it is fundamental to understand what sets the time for timed responses such as a motor action or an endocrine oscillation.

It appears that sleep and interval timing share a common destiny as they are often studied as emerging properties of neuronal networks. For this reason, it is not surprising that the function of sleep, is thought to be associated to synaptic plasticity, as theorized by the latest functional model of sleep (Tononi and Cirelli, 2006). In both sleep (Tononi and Cirelli, 2006) and interval timing (Buonomano and Laje, 2010) the output units of the network rely on the development and stabilization of proper synaptic weights. Yet, the synaptic interplay between frontal cortex and subcortical neurons, such as striatal neurons, is important in both sleep-related memory consolidation and interval timing. Besides, these phenomena rely on specific patterns of "slow" oscillatory firings (Diekelmann and Born, 2010).

A number of studies has shown that sleep benefits memory consolidation (see Diekelmann and Born, 2010 for an extensive review). Beneficial effects of sleep on memory occur after a few minutes (Lahl et al., 2008), a few hours (Mednick et al., 2003; Tucker et al., 2006a,b; Korman et al., 2007; Nishida and Walker, 2007), or after a proper night of sleep (Pace-Schott et al., 2005; Stickgold, 2005; Stickgold and Walker, 2005a,b; Walker et al., 2005a,b; Marshall and Born, 2007). Of all the memory tasks that have been used in investigating learning and memory aspects of sleep, almost none of them have manipulated temporal variables, until recently. Lewis et al. (2011) and Lewis and Meck, (2011), by using a combination of psychophysics and neuroimaging techniques in human participants, have tested the hypothesis that sleep promotes consolidation of temporal information. The authors differentiated motor and perceptual timing in their task. Interestingly, they reported that brain sleep-wake states during retention modulates motor learning in motor areas, such as the supplementary motor area, the striatum, and the cerebellum, while perceptual timing activates the posterior hippocampus zone (Lewis et al., 2011). This new evidence is in agreement with an influential model of a sleep-dependent memory mechanism that involves slow-wave sleep (SWS; Diekelmann and Born, 2010). Cortical slow oscillations $(<1 \mathrm{~Hz})$ provide a timed electrophysiological mechanism of up-and down-states that pass memories from hippocampal temporary storage to neocortical long-term storage (Cheng et al., 2008, 2009; Molle and Born, 2011).

\section{CIRCADIAN CLOCK AND INTERVAL TIMING}

The circadian clock is represented, at the cellular level, by a well-known negative transcription/translation-based feedback 
loop that sets the oscillation of the so called "core clock genes." The brain contains a pacemaker-like structure, the suprachiasmatic nuclei (SCN) of the hypothalamus, that provides circadian rhythmicity to other peripheral organs.

Core clock elements in the cell are transcriptional factors that regulate interlocking positive/negative feedback loops (Ko and Takahashi, 2006; Reppert, 2006). In mammals, the positive loop starts when CLOCK and BMAL1 (two members of the bHLHPAS transcriptional factor family), form heterodimers and then translocate to the nucleus. Here this CLOCK:BMAL1 complex binds to E-box enhancers and promote the transcription of the Period (PER1-3) and the Cryptochrome (CRY1 and CRY2) genes. The resulting proteins form PER:CRY heterodimers that initiate the negative feedback loop. These PER:CRY complexes move back to the nucleus and inhibit the activity of CLOCK:BMAL1 resulting, then, in the repression of their own transcription. However, these are not the only regulatory loops. CLOCK:BMAL1 activates also the transcription of two retinoic acid-related orphan receptors (REV-ERBs and RORs) which regulate positively (RORs) and negatively (REV-ERBs) Bmall. To increase the complexity of the circadian clock, a number of post-translational mechanisms were shown to regulate clock genes (LopezMolina et al., 1997; Lowrey and Takahashi, 2000; Lowrey et al., 2000). This whole clock mechanism of activators and repressors represent an oscillatory mechanism that is necessary to coordinate circadian rhythms. However, a remarkable causal relation exists between molecular oscillations and neural activity. An important property of neurons in the SCN of the hypothalamus is their ability to generate, endogenously, action potentials which oscillate throughout day and night (Albus et al., 2002; Schaap et al., 2003; Yamaguchi et al., 2003; Kuhlman and McMahon, 2006; Ko et al., 2009). Neurons are active for 4-6 h during the day and inactive during the night. During the daily active state their responses to excitatory inputs are remarkably reduced while at night they become responsive again. A combination of ion channels regulates membrane currents that maintain the spontaneous circadian activity of SCN neurons (Colwell, 2011). The silencing of SCN neuronal firing, at night, depends on a difference in membrane potential and this is mainly mediated by a hyperpolarizing potassium mechanisms (Kuhlman and McMahon, 2006).

A series of important studies, that have investigated the relation between neuronal activity and circadian molecular clock (Nitabach and Blau, 2002; Nitabach et al., 2002; Sheeba et al., 2008; Choi et al., 2009), has shown that synaptic activity and membrane potential are responsible for the oscillation of core clock genes. This has dramatically changed the general assumption that a molecular clock drives the activity of clock neurons. Nitabach et al. (2002) were able to show, in Drosophila, that chronic hyperpolarization silenced circadian neurons and interrupted the circadian behavioral rhythmicity and the expression of PER and TIM proteins. This testifies that neural activity regulates circadian molecular clock. Another component that plays a crucial role in clock gene expression is represented by the levels of $\mathrm{Ca}^{2+}$. The resting levels of $\mathrm{Ca}^{2+}$ during the day, when the SCN neurons are more active, are doubled if compared with the night inactivity (Colwell, 2000). $\mathrm{Ca}^{2+}$ is thought to be responsible also for another important aspect of circadian rhythms: the phase-response curve (Colwell, 2011). Indeed, the response to an environmental input (e.g., light stimulation) differs according to the specific phase of the circadian cycle.

There are several reasons to investigate associations between interval timing and circadian rhythms. A series of studies contravenes the assumption that interval timing depends on a linear accumulator but, instead, indicates an oscillatory-like mechanisms (Crystal, 1999, 2003; Crystal and Baramidze, 2007; Gu et al., 2011). Thus, it is reasonable to investigate if shared mechanisms between short (seconds-to-minutes) and long (circadian) timed responses occur. Several studies have concluded for a close relationship between interval timing and circadian rhythms. For example, it has been shown that circadian rhythms change the perception for short intervals (Pfaff, 1968; Aschoff, 1998a,b; Nakajima et al., 1998; Morofushi et al., 2001) and that in conditions of temporal isolation (Aschoff, 1998) the time estimation co-varies with their circadian period. Furthermore, in drosophila circadian mutants it is present a deficit for short-interval timing (Kyriacou and Hall, 1980). Yet, dopamine (DA) mechanisms and motivated behaviors are strongly associated with both interval timing (Meck, 2006a,b; Agostino et al., 2011) and circadian clock (Albrecht, 2011).SCN projects toward brain areas within the DA circuitries and which mediate reward-related behaviors.

Other studies, instead, suggested that circadian clock mechanisms are independent of interval timing. This conclusion was driven by SCN lesion studies (Lewis et al., 2003) and by investigations of interval timing in circadian mutants (Cordes and Gallistel, 2008; Papachristos et al., 2011). I shall argue that conclusions driven by lesions restricted to SCN should not be generalized to the molecular level. For example, studies in mice of SCN lesions that lead to arrhythmic circadian behaviors, but did not affect sleep homeostasis (Easton et al., 2004; Larkin et al., 2004), have supported, for a long time, the idea that the two processes (circadian and homeostatic) were independent. However, at a molecular level, we now know that several circadian genes play a role in sleep (Tucci and Nolan, 2010).

Regarding the phenotyping of interval timing in circadian mouse mutants, Cordes and Gallistel (2008) have reported that Clock has no abnormal consequences in the peakinterval procedure in mice. In this study CLOCK-KO mice were used instead of the CLOCK mutants that carry the single point mutations. Similar negative results in interval timing were obtained by Papachristos et al. (2011) in Cryl and Cry2 KO mice. Our critical argument to these studies is that, due to functional genomic redundancy, gene deletion models may not be able to reveal all the important functions of the gene. Paralog compensation among several clock functional genes has been reported (Debruyne et al. 2006; DeBruyne et al., 2007). It was shown that CLOCK-deficient mice present only mild circadian alterations and, thus, it is not essential for the circadian rhythms (Debruyne et al., 2006). A paralog of CLOCK, NSPAS2, has been proposed to dimerize with BIMAL1 and to work in the mouse forebrain as the clock molecular loop (Reick et al., 2001). NSPAS2 is particularly expressed in the cortex, hippocampus, striatum, amygdala, and thalamus (Garcia et al., 2000) and exerts an important role in sleep and behavior (Dudley et al., 2003). For all these reasons, I believe that further investigations in interval timing and circadian rhythms is required before we can roll out the hypothesis of an independency among these phenomena. 
EPIGENETICS AND BIOLOGICAL CLOCKS

Ultimately, the investigation of the genetic determinants that regulate biological clocks is growing in complexity. Epigenetic mechanisms set a number of temporal determinants for gene expression. The action of the genome seems to respond to a principle of modularity (Litvin et al., 2009) that refers to a functional model in which cellular states, determined by genetic variations and by extracellular stimulations, affect transcriptional responses (Litvin et al., 2009). In particular, a primary locus controls the states of the cell and then a secondary locus has an effect only in particular states. This is a very interesting model that implies a timed expression of specific gene-driven phenotypes. The temporal coding of genetic information depends on chromatin states that regulate gene transcription and functioning. Epigenetic marks can vary over periods of minutes to hours and they constitute fundamental mechanisms for learning and memory consolidation (Akbarian and Huang, 2009). Since approximately $10 \%$ of all mammalian transcripts present a circadian rhythmicity (Panda et al., 2002), an efficient chromatin remodeling must exist to ensure this rhythmic gene expression (Borrelli et al., 2008). A number of studies has demonstrated that methylation oscillates at clock gene promoters (Etchegaray et al., 2006) and rhythmic histone modification arises at promoter of clock-controlled genes (Etchegaray et al., 2003; Naruse et al., 2004; Ripperger and Schibler, 2006). Although these studies do not proof that specific epigenetic mechanisms, such as those involved in chromatin remodeling, are necessary for clock control, they demonstrate that transcription-permissive chromatin states occur at specific circadian times (Borrelli et al., 2008).

\section{CONCLUSION AND FUTURE DIRECTIONS}

Molecular elements play an important role in sleep, circadian rhythms, and interval timing. Thus, timing is coded at behavioral, physiological, genetic, and epigenetic level. An integrated investigation of these mechanisms represents the next frontier in developing models of coding and retaining of temporal information. The choice of animal models (e.g., the mouse), which carry single nucleotides mutations that translate into abnormal circadian phenotypes is preferred to deletion models. In future, it could be envisaged that interval-timing phenotypes will be largely employed in phenotype-based mutagenesis program (Nolan et al., 2000) and would have the potential to promote a new era of molecular discoveries, in interval timing, as we had for circadian clock. However, cognitive tests in mice present a number of restraints that make them unfeasible for such large-scale functional genomics enterprises. An easy solution to this impasse is the development of automated tests in home-cage. They have the advantage to increase the sample of observations, to reduce the time for training and to leave the animals undisturbed. Last, but not least, 24-h home-cage screens allow the integrated investigation of timing phenotypes at different time-scales.

\section{ACKNOWLEDGMENTS}

I thank Glenda Lassi for critical reading of the manuscript and for discussions. Support (to Valter Tucci) was provided by the European Commission FP7 Programme under project 223263 (PhenoScale).

\section{REFERENCES}

Agostino, P.V., Golombek, D. A., and Meck, W.H. (2011). Unwinding the molecular basis of interval and circadian timing. Front. Integr. Neurosci. 5:64. doi: 10.3389/ fnint.2011.00064

Akbarian, S., and Huang, H. S. (2009). Epigenetic regulation in human brain-focus on histone lysine methylation. Biol. Psychiatry 65, 198-203.

Albrecht, U. (2011). The circadian clock, reward, and memory. Front. Mol. Neurosci. 4:41. doi: 10.3389/ fnmol.2011.00041

Albus, H., Bonnefont, X., Chaves, I., Yasui, A., Doczy, J., van der Horst, G. T., and Meijer, J. H. (2002). Cryptochrome-deficient mice lack circadian electrical activity in the suprachiasmatic nuclei. Curr. Biol. 12, 1130-1133.

Aschoff, J. (1998a). Circadian parameters as individual characteristics. J. Biol. Rhythms 13, 123-131.

Aschoff, J. (1998b). Human perception of short and long time intervals: its correlation with body temperature and the duration of wake time. J. Biol. Rhythms 13, 437-442.

Borrelli, E., Nestler, E. J., Allis, C. D., and Sassone-Corsi, P. (2008). Decoding the epigenetic language of neuronal plasticity. Neuron 60, 961-974.

Buhusi, C. V., and Meck, W. H. (2005). What makes us tick? Functional and neural mechanisms of interval timing. Nat. Rev. Neurosci. 6, 755-765.

Buonomano, D. V., and Laje, R. (2010). Population clocks: motor timing with neural dynamics. Trends Cogn. Sci. (Regul. Ed.) 14, 520-527.

Cheng, R. K., Williams, C. L., and Meck, W. H. (2008). Oscillatory bands, neuronal synchrony and hippocampal function: implications of the effects of prenatal choline supplementation for sleepdependent memory consolidation. Brain Res. 1237, 176-194.
Cheng, R. K., Williams, C. L., and Meck, W. H. (2009). Neurophysiological mechanisms of sleep-dependent memory consolidation and its facilitation by prenatal choline supplementation. Chin. J. Physiol. 52, 223-235.

Choi, C., Fortin, J. P., McCarthy, E., Oksman, L., Kopin, A. S., and Nitabach, M. N. (2009). Cellular dissection of circadian peptide signals with genetically encoded membrane-tethered ligands. Curr. Biol. 19, 1167-1175.

Colwell, C. S. (2000). Circadian modulation of calcium levels in cells in the suprachiasmatic nucleus. Eur. J. Neurosci. 12, 571-576.

Colwell, C. S. (2011). Linking neural activity and molecular oscillations in the SCN. Nat. Rev. Neurosci. 12, 553-569.

Cordes, S., and Gallistel, C. R. (2008). Intact interval timing in circadian CLOCK mutants. Brain Res. 1227 120-127.

Coull, J. T., Cheng, R. K., and Meck, W. H. (2011). Neuroanatomical and neurochemical substrates of timing. Neuropsychopharmacology 36, 3-25.

Crystal, J. D. (1999). Systematic nonlinearities in the perception of temporal intervals. J. Exp. Psychol. Anim. Behav. Process. 25, 3-17.

Crystal, J. D. (2001). Circadian time perception. J. Exp. Psychol. Anim. Behav. Process. 27, 68-78.

Crystal, J. D. (2003). "Nonlinearities in sensitivity to time: implications for oscillator-based representations of interval circadian clocks," in Functional and Neural Mechanisms of Interval Timing, ed. W. H. Meck (Boca Raton, FL: CRC Press), 61-76.

Crystal, J. D. (2006a). Animal behavior: timing in the wild. Curr. Biol. 16, R252-R253.

Crystal, J. D. (2006b). Long-interval timing is based on a self-sustaining endogenous oscillator. Behav. Processes 72, 149-160.

Crystal, J. D., and Baramidze, G. T. (2007). Endogenous oscillations in short-interval timing. Behav. Processes 74, 152-158.

Debruyne, J. P., Noton, E., Lambert, C. M., Maywood, E. S., Weaver, D. R., and Reppert, S. M. (2006). A clock shock: mouse CLOCK is not required for circadian oscillator function. Neuron 50, 465-477.

DeBruyne, J. P., Weaver, D. R., and Reppert, S. M. (2007). CLOCK and NPAS2 have overlapping roles in the suprachiasmatic circadian clock. Nat. Neurosci. 10, 543-545.

Diekelmann, S., and Born, J. (2010). The memory function of sleep. Nat. Rev. Neurosci. 11, 114-126.

Dudley, C. A., Erbel-Sieler, C., Estill, S. J., Reick, M., Franken, P., Pitts, S., and McKnight, S. L. (2003). Altered patterns of sleep and behavioral adaptability in NPAS2-deficient mice. Science 301, 379-383.

Easton, A., Meerlo, P., Bergmann, B., and Turek, F. W. (2004). The suprachiasmatic nucleus regulates sleep timing and amount in mice. Sleep 27, 1307-1318.

Etchegaray, J. P., Lee, C., Wade, P. A., and Reppert, S. M. (2003). Rhythmic histone acetylation underlies transcription in the mammalian circadian clock. Nature 421177-421182.

Etchegaray, J. P., Yang, X., DeBruyne, J. P., Peters, A. H., Weaver, D. R., Jenuwein, T., and Reppert, S. M. (2006) The polycomb group protein EZH2 is required for mammalian circadian clock function. J. Biol. Chem. 281, 21209-21215.

Franken, P., and Dijk, D. J. (2009). Circadian clock genes and sleep homeostasis. Eur. J. Neurosci. 29, 1820-1829. 
Garcia, J.A., Zhang, D., Estill, S. J., Michnoff, C., Rutter, J., Reick, M., Scott, K., Diaz-Arrastia, R., and McKnight, S. L. (2000). Impaired cued and contextual memory in NPAS2-deficient mice. Science 288, 2226-2230.

Gu, B. M., Yin, B., Cheng, R. K., and Meck, W. H. (2011). Quinpirole-induced sensitization to noisy/sparse periodic input: temporal synchronization as a component of obsessive-compulsive disorder. Neuroscience 179, 143-150.

Hinton, S. C., and Meck, W. H. (1997). The 'internal clocks' of circadian and interval timing. Endeavour 21, 82-87.

Ko, C. H., and Takahashi, J. S. (2006). Molecular components of the mammalian circadian clock. Hum. Mol. Genet. 15 Spec No 2, R271-R277.

Ko, G. Y., Shi, L., and Ko, M. L. (2009). Circadian regulation of ion channels and their functions. J. Neurochem. 110, 1150-1169.

Korman, M., Doyon, J., Doljansky, J., Carrier, J., Dagan, Y., and Karni, A. (2007). Daytime sleep condenses the time course of motor memory consolidation. Nat. Neurosci. 10, 1206-1213.

Krueger, J. M. (2010). "What exactly is it that sleeps? The evolution, regulation and organization of an emergent network property," in Evolution of Sleep: Phylogenetic and Functional Perspectives, eds P. McNamara, R. A. Barton, and C. L. Nunn (New York: Cambridge University Press), 86-106.

Kuhlman, S. J., and McMahon, D. G. (2006). Encoding the ins and outs of circadian pacemaking. J. Biol. Rhythms $21,470-481$.

Kyriacou, C. P., and Hall, J. C. (1980). Circadian rhythm mutations in Drosophila melanogaster affect shortterm fluctuations in the male's courtship song. Proc. Natl. Acad. Sci. U.S.A. 77, 6729-6733.

Lahl, O., Wispel, C., Willigens, B., and Pietrowsky, R. (2008). An ultra short episode of sleep is sufficient to promote declarative memory performance. J. Sleep Res. 17, 3-10.

Larkin, J. E., Yokogawa, T., Heller, H. C., Franken, P., and Ruby, N. F. (2004). Homeostatic regulation of sleep in arrhythmic Siberian hamsters. Am. J. Physiol. Regul. Integr. Comp. Physiol. 287, R104-R111.

Lewis, P.A., Couch, T.J., and Walker, M.P. (2011). Keeping time in your sleep: overnight consolidation of temporal rhythm. Neuropsychologia 49, 115-123.

Lewis, P. A., and Meck, W. H. (2011). Does sleep contribute to degeneracy in neural timing? Psychologist (in press).

Lewis, P.A., Miall, R. C., Daan, S., and Kacelnik, A. (2003). Interval timing in mice does not rely upon the circadian pacemaker. Neurosci. Lett. 348, 131-134.

Litvin, O., Causton, H. C., Chen, B. J., and Pe'er, D. (2009). Modularity and interactions in the genetics of gene expression. Proc. Natl. Acad. Sci. U.S.A. 106, 6441-6446.

Lopez-Molina, L., Conquet, F., Dubois-Dauphin, M., and Schibler, U. (1997). The DBP gene is expressed according to a circadian rhythm in the suprachiasmatic nucleus and influences circadian behavior. EMBO J. 16, 6762-6771.

Lowrey, P. L., Shimomura, K., Antoch, M. P., Yamazaki, S., Zemenides, P. D., Ralph, M. R., Menaker, M., and Takahashi, J. S. (2000). Positional syntenic cloning and functional characterization of the mammalian circadian mutation tau. Science 288, 483-492.

Lowrey, P. L., and Takahashi, J. S. (2000). Genetics of the mammalian circadian system: photic entrain- ment, circadian pacemaker mechanisms, and posttranslational regulation. Annu. Rev. Genet. 34, 533-562.

Marshall, L., and Born, J. (2007). The contribution of sleep to hippocampus-dependent memory consolidation. Trends Cogn. Sci. (Regul. Ed.) 11, 442-450.

Meck, W. H. (2006a). Frontal cortex lesions eliminate the clock speed effect of dopaminergic drugs on interval timing. Brain Res. 1108, 157-167.

Meck,W.H. (2006b). Neuroanatomical localization of an internal clock: a functional link between mesolimbic, nigrostriatal, and mesocortical dopaminergic systems. Brain Res. 1109, 93-107.

Mednick, S., Nakayama, K., and Stickgold, R. (2003). Sleep-dependent learning: a nap is as good as a night. Nat. Neurosci. 6, 697-698.

Molle, M., and Born, J. (2011). Slow oscillations orchestrating fast oscillations and memory consolidation. Prog. Brain Res. 193, 93-110.

Morofushi, M., Shinohara, K., and Kimura, F. (2001). Menstrual and circadian variations in time perception in healthy women and women with premenstrual syndrome. Neurosci. Res. 41, 339-344.

Nakajima, T., Uchiyama, M., Enomoto, T., Shibui, K., Ishibashi, K., Kanno, O., and Okawa, M. (1998). Human time production under constant routine. Psychiatry Clin. Neurosci. 52 240-241.

Naruse, Y., Oh-Hashi, K., Iijima, N.,Naruse, M., Yoshioka, H., and Tanaka, M. (2004). Circadian and lightinduced transcription of clock gene Per1 depends on histone acetylation and deacetylation. Mol. Cell Biol. 24, 6278-6287.

Nishida, M., and Walker, M. P. (2007). Daytime naps, motor memory consolidation and regionally specific sleep spindles. PLoS ONE 2, e341. doi: 10.1371/journal.pone.0000341

Nitabach, M. N., and Blau, J. (2002). Cellular clockwork. Nat. Genet. 32, 559-560.

Nitabach, M. N., Blau, J., and Holmes, T. C. (2002) Electrical silencing of Drosophila pacemaker neurons stops the free-running circadian clock. Cell 109, 485-495.

Nolan, P. M., Peters, J., Strivens, M., Rogers, D., Hagan, J., Spurr, N., Gray, I. C., Vizor, L., Brooker, D., Whitehill, E., Washbourne, R., Hough, T., Greenaway, S., Hewitt, M., Liu, X., McCormack, S., Pickford, K., Selley, R., Wells, C., Tymowska-Lalanne, Z., Roby, P., Glenister, P., Thornton, C., Thaung, C., Stevenson, J. A., Arkell, R., Mburu, P., Hardisty, R., Kiernan, A., Erven, A., Steel, K. P., Voegeling, S., Guenet, J. L., Nickols, C., Sadri, R., Nasse, M., Isaacs, A., Davies, K., Browne, M., Fisher, E. M., Martin, J., Rastan, S., Brown, S. D., and Hunter, J. (2000). A systematic, genome-wide, phenotype-driven mutagenesis programme for gene function studies in the mouse. Nat. Genet. 25, 440-443.

Pace-Schott, E. F., Stickgold, R., Muzur, A., Wigren, P. E., Ward, A. S., Hart, C. L., Walker, M., Edgar, C., and Hobson, J. A. (2005). Cognitive performance by humans during a smoked cocaine binge-abstinence cycle. Am. J. Drug Alcohol Abuse 31, 571-591.

Panda, S., Antoch, M. P., Miller, B. H., Su, A. I., Schook, A. B., Straume, M., Schultz, P. G., Kay, S. A., Takahashi, J. S., and Hogenesch, J. B. (2002). Coordinated transcription of key pathways in the mouse by the circadian clock. Cell 109, 307-320.

Papachristos, E. B., Jacobs, E. H., and Elgersma, Y. (2011) Interval timing is intact in arrhythmic Cry1/Cry2deficient mice. J. Biol. Rhythms 26, 305-313.
Pfaff, D. (1968). Effects of temperature and time of day on time judgments. J. Exp. Psychol. 76, 419-422.

Reick, M., Garcia, J. A., Dudley, C., and McKnight, S. L. (2001). NPAS2: an analog of clock operative in the mammalian forebrain. Science 293, 506-509.

Reppert, S. M. (2006). A colorful model of the circadian clock. Cell 124, 233-236.

Ripperger, J. A., and Schibler, U. (2006). Rhythmic CLOCK-BMAL1 binding to multiple E-box motifs drives circadian Dbp transcription and chromatin transitions. Nat. Genet. 38, 369-374.

Schaap, J., Albus, H., VanderLeest, H. T., Eilers, P. H., Détári, L., and Meijer, J. H. (2003). Heterogeneity of rhythmic suprachiasmatic nucleus neurons: implications for circadian waveform and photoperiodic encoding. Proc. Natl. Acad. Sci. USA 100 15994-15999.

Sheeba, V., Gu, H., Sharma, V. K., O’Dowd, D. K., and Holmes, T.C. (2008). Circadian- and light-dependent regulation of resting membrane potential and spontaneous action potential firing of Drosophila circadian pacemaker neurons. J. Neurophysiol. 99, 976-988.

Stickgold, R. (2005). Sleep-dependent memory consolidation. Nature 437, 1272-1278.

Stickgold, R., and Walker, M. P. (2005a). Memory consolidation and reconsolidation: what is the role of sleep? Trends Neurosci. 28, 408-415.

Stickgold, R., and Walker, M. P. (2005b). Sleep and memory: the ongoing debate. Sleep 28, 1225-1227.

Tononi, G., and Cirelli, C. (2006). Sleep function and synaptic homeostasis. Sleep Med. Rev. 10, 49-62.

Tucci, V., and Nolan, P. M. (2010). “Toward an understanding of the function of sleep: new insights from mouse genetics," in Evolution of Sleep: Phylogenetic and Functional Perspectives, eds P. McNamara, R. A. Barton, and C. L. Nunn (New York: Cambridge University Press), 218-237.

Tucker, M. A., Hirota, Y., Wamsley, E. J., Lau, H., Chaklader, A., and Fishbein, W. (2006a). A daytime nap containing solely non-REM sleep enhances declarative but not procedural memory. Neurobiol. Learn. Mem. 86, 241-247.

Tucker, P., Lombardi, D., Smith, L., and Folkard, S. (2006b). The impact of rest breaks on temporal trends in injury risk. Chronobiol. Int. 23, 1423-1434.

Walker, M. P., Stickgold, R., Alsop, D., Gaab, N., and Schlaug, G. (2005a). Sleep-dependent motor memory plasticity in the human brain. Neuroscience 133, 911-917.

Walker, M. P., Stickgold, R., Jolesz, F. A., and Yoo, S. S. (2005b). The functional anatomy of sleep-dependent visual skill learning. Cereb. Cortex 15, 1666-1675.

Yamaguchi, S., Isejima, H., Matsuo, T., Okura, R., Yagita, K., Kobayashi, M., and Okamura, H. (2003). Synchronization of cellular clocks in the suprachiasmatic nucleus. Science 302, 1408-1412.

Received: 15 December 2011; accepted: 15 December 2011 published online: 04 January 2012.

Citation: Tucci V (2012) Sleep, circadian rhythms, and interval timing: evolutionary strategies to time information. Front. Integr. Neurosci. 5:92. doi: 10.3389/fnint.2011.00092 Copyright $\odot 2012$ Tucci. This is an open-access article distributed under the terms of the Creative Commons Attribution Non Commercial License, which permits non-commercial use, distribution, and reproduction in other forums, provided the original authors and source are credited. 